\title{
Molecular and Evolutionary Determinants of Bacteriophage Host Range
}

\author{
Patrick A. de Jonge, ${ }^{1,2}$ Franklin L. Nobrega, ${ }^{2}$ Stan J.J. Brouns, ${ }^{2,3,5}$ and Bas E. Dutilh $\mathbb{D}^{1,4,5, \star}$
}

The host range of a bacteriophage is the taxonomic diversity of hosts it can successfully infect. Host range, one of the central traits to understand in phages, is determined by a range of molecular interactions between phage and host throughout the infection cycle. While many well studied model phages seem to exhibit a narrow host range, recent ecological and metagenomics studies indicate that phages may have specificities that range from narrow to broad. There is a growing body of studies on the molecular mechanisms that enable phages to infect multiple hosts. These mechanisms, and their evolution, are of considerable importance to understanding phage ecology and the various clinical, industrial, and biotechnological applications of phage. Here we review knowledge of the molecular mechanisms that determine host range, provide a framework defining broad host range in an evolutionary context, and highlight areas for additional research.

\section{The Importance of Host Range}

One hundred years after their initial discovery, research into bacteriophages - viruses that infect bacteria - is undergoing a surge of interest. This increased interest is driven by a new appreciation of the ecological impact of phages [1], their potential applications [2], and their role in human health $[3,4]$. For example, metagenomic surveys have uncovered a wealth of viral diversity in natural and human-associated samples [4-6]; novel applications of phages are being developed (Box 1); and novel theoretical models have increased the focus on the ecoevolutionary roles of phages in natural microbial systems [7,8] (Box 2). To place the plethora of new information into context, it is crucial to understand the interactions that phages have with their hosts. Host interaction has implications for how phages control microbial populations $[9,10]$, how they facilitate horizontal gene transfer [11], and how they can be deployed in clinical and industrial applications (Box 1).

Currently, the majority of phages and their host interactions remain unstudied in the laboratory because (i) many host bacteria remain uncultivable, and (ii) as we will show, the techniques used for isolating and studying phages are biased [12]. For phages that have been tested, infection assays have shown that host range spans a wide continuum, from extremely narrow to broad [13]. Recent studies of newly isolated marine broad-host-range phage (see Glossary) lineages (e.g., $[14,15])$ and community-wide single-cell metagenomics efforts of extreme environments [16] indicate that broad-host-range phages are potentially widely distributed in natural environments. This is supported by a number of recent metagenomics efforts that have sought to link viral genomes to their hosts [17,18], suggesting that a wide variety of host ranges occur in nature (Figure 1). Such complementary, and sometimes contrasting, lines of evidence - combined with the importance of phage host range and host-range variation for understanding phage biology, microbial ecology, and phage applications - justify studying the specific molecular mechanisms that allow phages to infect multiple hosts.

\begin{abstract}
Highlights
Broad-host-range phages are more common in nature than previously thought, and most environments have a variety of narrow-range and broad-host-range phages.

In phenotypic broad-host-range phages, individual phage particles can infect multiple hosts. In genotypic broad-host-range phages, individual particles infect only one host, but specific mutations enable rapid host switching between generations, leading to a broad-host-range quasispecies.

Broad-host-range phages, engineered or natural, are useful for treating multiple-strain infections, but phage therapy is dependent on a full understanding of host-range evolution.

The widespread existence of narrowhost-range phages among those studied is at least partly caused by biases in phage-isolation methods.

Ecological parameters, such as host density and host diversity, probably determine the likelihood and richness of broad-hostrange phages in a given environment.
\end{abstract}

${ }^{1}$ Theoretical Biology and Bioinformatics, Utrecht University, Padualaan 83584 $\mathrm{CH}$ Utrecht, The Netherlands ${ }^{2}$ Department of Bionanoscience, Kavli Institute of Nanoscience, Delft University of Technology, Van der Maasweg 9 2629 HZ, Delft, The Netherlands ${ }^{3}$ Laboratory for Microbiology, Wageningen University, Stippeneng 4 6708 WE, Wageningen, The Netherlands ${ }^{4}$ Centre for Molecular and Biomolecular Informatics, Radboud University Medical Centre, Geert Grooteplein Zuid 26-28, 6525GA Nijmegen, The Netherlands ${ }^{5}$ These authors made equal contributions

${ }^{*}$ Correspondence: bedutilh@gmail.com (B.E. Dutilh). 


\section{Box 1. Host Range in Phage Applications}

Several applications critically depend on the host specificity of phages. Phage typing utilizes the specific nature of phage-host interactions by generating a phage-susceptibility pattern unique to a singular bacterial pathogen [94]. Such patterns can subsequently be used to distinguish between bacteria and track different pathogenic strains. As identifying pathogens using phage typing is wholly dependent on the specificity of the lysis pattern, phages with highly stable host affinity are essential.

With the rise of antibiotic resistance among pathogens worldwide, phage therapy is increasingly considered as a viable alternative for application both in medicine and in the food industry [88,95]. Although the idea behind phage therapy is as old as phage biology, recent years have seen an impressive revival (e.g., $[96,97])$. In phage therapy, phage hostspecificity is a double-edged sword. On the one hand, narrow-host-range phages preserve the natural microbial flora and lower the probability of developing community-wide resistance [98,99]. On the other hand, such phages may need to be isolated for each target strain, increasing labour requirements for their isolation and validation. Moreover, pathogens may readily evolve resistance to individual phages, so infections need to be treated with phage cocktails where each of the members are to be validated [100]. Broad-host-range phages may be a promising solution, but before this can be developed the evolutionary stability of phage-host interactions needs to be established.

Efforts to control microbial community composition further hinge on an accurate understanding of phage host range and its evolution. For example, temperate phages have been used to spread sensitivity to antibiotics in E. coli $[101,102]$ and to deliver a CRISPR-Cas9 system designed to specifically kill pathogenic bacteria [103]. In such applications, broad host range was found to be an asset when targeting specific bacteria in heterogeneous environments [9].

\section{Defining Host Range}

Like all aspects of phage biology, host range can be understood only in the context of the phage life cycle. The best described are the lytic and temperate life cycles. Upon infection, obligately Iytic phages rapidly produce offspring and lyse the host, while temperate phages may first integrate their genome into the host genome, creating a lysogen, - until stressors prompt them to become lytic. Other life cycles, such as chronic infections and pseudolysogeny, are less widely described, and their ubiquity remains unknown $[19,20]$.

The lytic phase of the phage life cycle includes four stages. First, a phage encounters a suitable host, attaches to its surface, and inserts its genomic material into the host cell. Second, the

\section{Box 2. Ecoevolutionary Models of Phage Host Range}

The main tools for studying the ecological and evolutionary significance of the host-range continuum include theoretical models [104], statistical analyses [105], and coevolutionary experiments [106]. These studies have led to two major competing models of phage-host coevolution: arms race dynamics (ARD) and fluctuating state dynamics (FSD) [107].

In ARD, host and phage iteratively evolve new resistance and infectivity, respectively, while maintaining resistance and infectivity to ancestral antagonists. Thus, over time, phages evolve a broader host range which includes progressively more resistant hosts. When depicted in a matrix, the resulting interaction network is nested [105], where the more specialist phages iteratively infect subsets of the hosts relative to the more generalist phages. Antagonistic pleiotropy of infective traits in phages would keep phages with universal host ranges from evolving in such a system [108].

In FSD, phage-host interactions remain highly specific. When hosts evolve novel resistance, phages overcome this to maintain infectivity, but in the process they lose the ability to infect ancestral hosts. In a similar fashion, hosts are only resistant against co-occurring phage strains but not against ancestral strains. This causes a fluctuating selection between highly specific host and phage genotypes, an extreme case being a monogamous network of phage-host interactions where one phage infects one host [104]. However, modular networks may be more likely in heterogeneous natural environments, where phages and hosts interact in subsets.

Small-scale, local networks often show nested structures [105], while modularity becomes evident at larger scales $[109,110]$. Host range is likely bounded by the phylogenetic, geographical, and ecological distance between a phage and its potential hosts [111], phages not always being adapted to local hosts [112]. Phages have been observed to be specific not so much to a species but to a locale [106,113], with different locales containing different frequencies of broad-host-range phages [114]. Finally, interaction networks imperfectly reflect coevolutionary dynamics [115], so it is clear that many mechanisms drive phage evolution [35] and much remains to be discovered.

\section{Glossary}

Antagonistic pleiotropy: pleiotropy is a phenomenon where one gene regulates multiple phenotypic traits. In antagonistic pleiotropy, thought to be common in specialized genomes, one mutation is beneficial to one trait and detrimental to another trait. For example, a given mutation may increase a phage's host range but decrease its infectivity on each individual host.

Broad-host-range phage: a phage that infects multiple distinct hosts within one genus (e.g., Bordetella phage BPP-1) or in multiple genera (e.g., phage SN-T).

Lysogen: a bacterium containing a prophage that is integrated into its genome. Specific prophage genes can affect the phenotype of lysogens. Various environmental factors can induce a prophage to become lytic.

Lytic phage: a phage that, upon infection, hijacks host cell mechanisms to produce new phage particles and eventually lyses the host to release the produced progeny. Obligately Iytic phages are always lytic, as opposed to phages that can become lytic or temperate in different environments.

Monovalent phage: recognizes a single receptor on the host surface. Polyvalent phage: recognizes multiple different receptor molecules. Quasispecies: a group of biological entities (like viruses) that exhibit high mutation rates, leading to a cloud of very closely related genotypes with specific evolutionary properties.

Receptor-binding protein (RBP): phage proteins that bind to a receptor on the host cell surface as the first step of infection.

Temperate phage: a phage that integrates its genome into that of its host, forming a lysogen, and replicating with the host genome. 


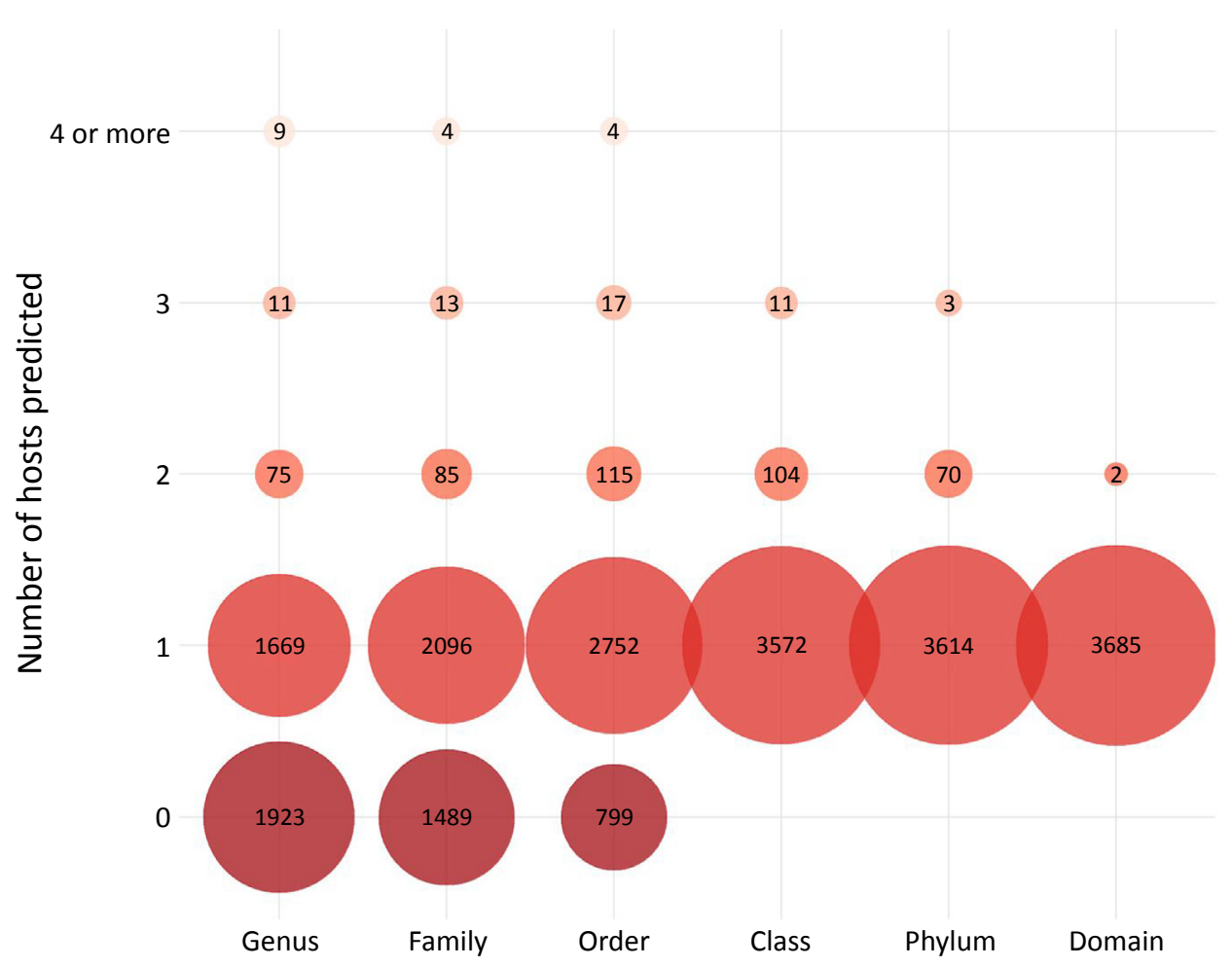

Host taxonomic level

Figure 1. Meta-analysis Shows Diversity Predicted Host Ranges of Phages Identified by Metagenomics. A recent metagenomic virus discovery study [1] used various signals to predict the host range of 15280 detected viruses, including genetic homology, oligonucleotide usage patterns, clustered regularly interspaced short palindromic repeats (CRISPR) spacer matches, and tRNAs. Hosts were predicted for 3687 out of 15280 viruses. It remains difficult to make accurate host predictions, especially at lower host taxonomic levels: 1764 hosts were predicted at the genus level, and no species- or strain-level host predictions were made. Each column in the graph adds up to all 3687 viral sequences for which at least one host prediction was made, for example, 75 viruses had hosts predicted in two different genera, while 13 viruses had hosts predicted in three different orders, etc. Similar host-range patterns were observed in another recent study [18].

phage bypasses host intracellular defences to establish a sustainable infection. Third, it replicates by hijacking resources of the host cell to produce progeny. Fourth, the phage progeny escape from the host cell into the environment, restarting the cycle. Many studies into the molecular mechanisms of broad-host-range phages focus on the attachment stage. For example, monovalent phages (i.e., those that bind to a single receptor) are more likely to have a narrow host range, while polyvalent phages (i.e., those that can bind multiple different receptors) may be able to infect more diverse hosts. However, each step in the life cycle involves circumventing defences and may require molecular and regulatory systems that are general enough to interact with multiple hosts. As we discuss below, host-range modulation may depend on adaptations in the host receptor-binding protein (RBP), but also on proteins that play a role in other life cycle stages. Together, we review current knowledge on the molecular mechanisms that allow broad-host-range phages to infect multiple hosts and discuss the evolutionary context of the host-range phenotype.

Despite the importance of understanding host range in the context of phage ecology, and in potential phage applications, what constitutes 'broad' versus 'narrow' is not clearly delineated. 
The depth and breadth of bacterial and viral diversity, and difficulties in clearly delineating phage and bacterial taxonomy [21,22], make the formulation of specific definitions problematic. Also, both experimental and computational assessments of phage host range have their limitations [23], potentially leading to mislabelled host range. Finally, phages are notoriously quick to mutate and evolve, and under the right circumstances phages may switch from a narrow to a broad host range, and back [24].

For the purpose of this review, we define narrow-host-range phages as those that can complete their life cycle in only one host (Figure 2A), and broad-host-range phages as those that can complete their life cycle in multiple taxonomically distinct hosts. There is no clear categorical distinction between different levels of the broad host range. Phages that infect multiple bacterial strains of the same species and those that infect bacteria from different genera are both said to have a broad host range, even though the latter range is obviously broader than the former. Here, where relevant, we specify at which level the multiple infected hosts are taxonomically related.

We further distinguish phenotypical and genotypical mechanisms facilitating broad host range. Phenotypical mechanisms involve individual broad-host-range phage particles that are capable of

\section{(A) Narrow host range}

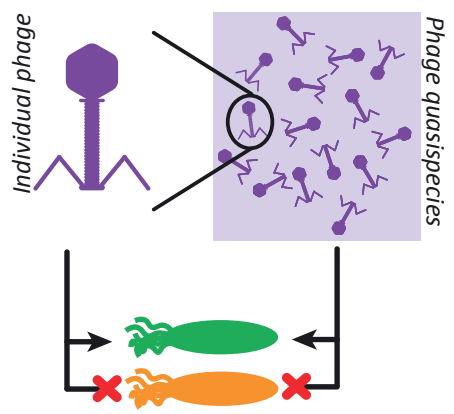

(B) Broad host range

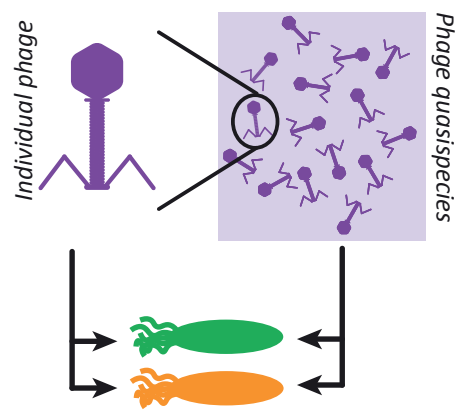

(C) Host switching

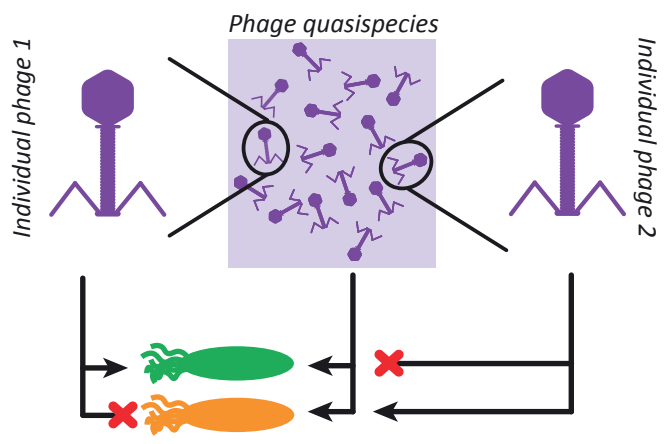

Trends in Microbiology

Figure 2. Subdivision of Bacteriophages Based on Host Range. Phages can be divided into three basic groups based on their host range and the mechanism used to achieve it. (A) Narrow-host-range phages infect only one host (most studied phages). (B) Broad-host-range phage species infect multiple hosts, an ability present in every individual phage in the species (e.g., SP6 [26]). (C) Host switching is a grey area between narrow and broad host range. Here, the quasispecies as a whole can infect multiple hosts, but each individual particle has the capability to infect only a single host (e.g., BPP-1 [37]). 
infecting multiple hosts (e.g., Phi92 [25] or SP6 [26], Figure 2B). Genotypical mechanisms involve host-switching phages where the entire phage quasispecies can infect multiple hosts, while individual particles can infect only one host (Figure 2C). The broad host range is thus the result of changes in the individual phage genotype. This difference may be important when formulating theoretical models to interpret microbial and viral dynamics when such data are available (Box 2).

In the following sections, we first review the dedicated mechanisms that broad-host-range phages possess at every stage in their life cycle, followed by a discussion of the experimental techniques that have been developed to isolate and study such phages. Together, we provide a comprehensive review of the current state of knowledge about phage host range and hope to stimulate interest in this important topic.

\section{Natural and Engineered Surface-Adhesion Mechanisms of Broad-Host- Range Phages}

The initial interaction between phage and host is a chance encounter; in many studied phages it is followed by a two-step process of reversible and irreversible binding of the RBPs to host surface receptors [27]. As surface structures are among the most variable elements of a microbial cell, and nearly every surface structure can serve as phage receptor [28], the initial stage of phage infection is an important determinant of phage host range. Monovalent broadhost-range phages may target structures on the host cell wall that are conserved between different hosts, such as some Salmonella phages that target the sugar cores of lipopolysaccharides in multiple hosts [29]. Polyvalent broad-host-range phages encode mechanisms for binding multiple different receptors. The following discusses the various phenotypic and genotypic mechanisms that allow phages to bind to the cell walls of multiple hosts, as well as ways in which these mechanisms can be exploited to engineer broad-host-range phages.

Specific Phage Particles and a Broad-Host-Range Quasispecies

Phage-host coevolution studies have shown that host specificity can be altered through mutations in the RBP gene [30-32] as well as in other genes [33]. Phage host-range mutations can be caused by as little as one point mutation [34], this limited number allowing reversibility and repeatability in host-range evolution [31] (Figure 3A). However, most mutations do not lead to a change in host range [35] due to the more constrained nature of gain-of-function mutations in the phage versus loss-of-function mutations in the host [30].

Polyvalent phage lineages with dual-receptor specificity can result from RBP mutations. For example, the narrow-host-range enterobacteria phage $\lambda$ can evolve into a broad-host-range phage capable of infecting hosts from different genera by binding to either OmpF or to the ancestral LamB protein receptors [31]. Further coevolution led to specialisation of this broadhost-range phage to either OmpF- or LamB-specific monovalent phages, even when both receptors were present in the environment [24]. Moreover, the genetically uniform broad-hostrange lineage also seemed to consist of two subpopulations preferring either LamB or OmpF, an effect hypothesized to be caused by differences in RBP folding [36]. These results show that the transition between monovalence and polyvalence can be readily made.

Some phages possess molecular mechanisms that allow for targeted genetic diversification of RBP, thereby allowing controlled host switching. In the Bordetella phage BPP-1 [37], a reverse transcriptase allows for reverse transcription and adenosine-specific mutagenesis of a template region in its genome, which is then used to substitute the $3^{\prime}$-end hypervariable domain of the RBP-encoding $m t d$ gene (Figure 3B). Another strategy was found in enterobacteria phage $\mathrm{T} 4$, where duplication of hypervariable domains flanked by a $\mathrm{GxH} \mathrm{xH}$ motif expanded its host 
(A) Point mutations

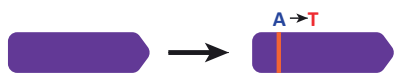

(C) His-box duplication
(B) Reverse transcriptase

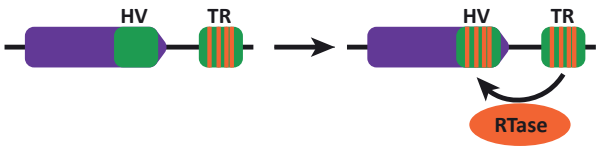

(D) Reversible RBP segment Reversible segment

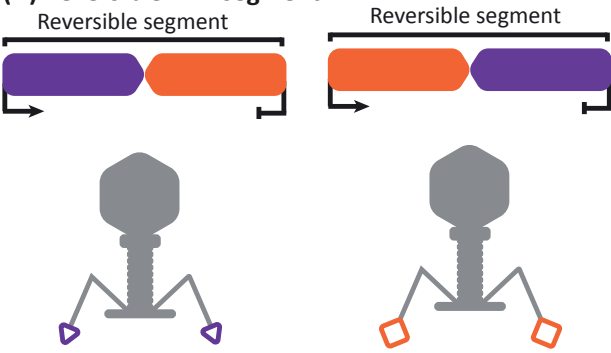

Trends in Microbiology

Figure 3. Mutational Strategies for Broadening or Switching Host Range. Some phages are known to employ methods to mutate their receptor-binding proteins (RBPs) so as to change receptor specificity. While each individual virion is monovalent, these phages represent a polyvalent quasispecies. (A) Point mutations in the gene encoding the RBP may alter the structure of the protein. These point mutations are random, although convergent evolution has been observed (examples in main text). (B) The polyvalent Bordetella phage BPP-1 alters its host specificity through a reverse transcriptase-mediated mechanism. This protein introduces a combination of specific mutations to a template region (TR) upstream of the RBP. This subsequently replaces the hypervariable region (HV) at the $3^{\prime}$ end of the gene [37]. (C) In phage $\mathrm{T} 4$, variable sections of the RBPs are flanked by GxHxH motifs called His-boxes. Duplication of these His-boxes changes RBP specificity [38]. (D) Phages Mu and P1 have two RBPs with different specificities organised in a reversible genome segment. This segment can be flipped around in the prophage state, thereby determining which RBP is transcribed [41].

range from Escherichia coli to include Yersinia pseudotuberculosis, a different host within the order Enterobacterales [38] (Figure 3C). The discovery of such hypervariable regions in diverse phage contigs assembled from human faecal metagenomes suggests that the mechanism may be widespread [39]. For example, a similar system to the one in BPP-1 has been found in a taxonomically distinct intraterrestrial archaeal virus, ANMV-1 [40].

Finally, the genome in some phages encodes multiple RBPs, but only one RBP is expressed at any given time. Enterobacterial phages Mu and P1 encode two RBPs with distinct specificities. The orientation of a reversible genomic segment encoding both RBPs determines which RBP is expressed - and therewith the specificity of the phage particles (Figure 3D) [41].

\section{Broad-Host-Range Phage Particles}

Recent cryoelectron tomography (cryo-ET) studies have revealed polyvalent phages that simultaneously express multiple RBPs. Salmonella phage SP6 can infect multiple S. enterica subsp. enterica serovars by expressing two RBPs on a V-shaped structure [26] that swivels to change tailspike orientation depending on the host species encountered (Figure 4A). Similarly, E. coli phages DT57C and DT571/2 encode dual RBPs that are hypothesized to branch out from the phage tail fibre, possibly in a similar fashion to SP6 RBPs [42]. This mechanism also occurs in phages that infect Gram-positive bacteria, such as Staphylococcus phage $\varphi$ SA012, which uses a main RBP to bind to the teichoic acid core of some Staphylococcus aureus strains and a second RBP to bind an $\alpha-N$-acetylglucosamine moiety in $S$. aureus strains where the teichoic acid core is unavailable due to glycosylation [29].

Enterobacteria phage $\Phi 92$ encodes no fewer than five RBP genes for infection of several different E. coli and Salmonella strains [25]. Cryo-ET showed at least three distinct RBPs 


\section{(A) V-shaped tail spike}

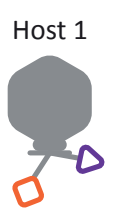

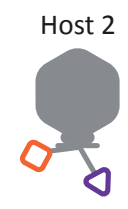

(B) Radial RBP

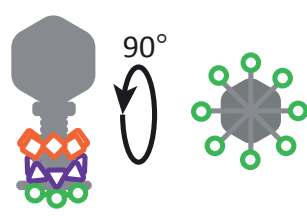

Trends in Microbiology
Figure 4. Phages Encoding Multiple Receptor-binding Proteins (RBPs) with Differing Specificity. Some phages encode and/or express multiple RBPs to allow binding to multiple receptors, thus representing polyvalent virions. (A) Phage SP6 possesses two RBPs at the end of a V-shaped tailspike. Changing the angle of the tailspike determines which RBP can bind to a receptor [26]. (B) Phage phi92 encodes at least five RBPs in its genome. At least three of these are expressed at the end of the tail fibre. It is unknown whether the remaining two RBPs are expressed [25].

radiating from the $\Phi 92$ baseplate, each in six-fold (Figure 4B). It remains unknown if the other two encoded RBP genes are functional or if different selections of the five RBPs are expressed at a time. These results show how recent developments in electron microscopy now allow the study of phage-host interactions in greater detail than before. Although only the SP6 and $\Phi 92$ mechanisms have been studied in detail, future studies will undoubtedly uncover other broadhost-range mechanisms in phages that encode multiple putative RBPs, such as Sinorhizobium phages $\Phi \mathrm{M9}$ and P10VF [43].

An intriguing alternative that allows phages to overcome receptor specificity without encoding multiple RBPs has been observed in Bacillus subtilis cultures infected with Bacillus phage SPP1 [44]. Phage-induced cell lysis promotes formation of membrane vesicles [45], some of which contain the SPP1 receptor. When such vesicles merge with resistant cells they may become transiently sensitive to the phage. While this might be a rare chance event, it could effectively increase the host range of monovalent phages.

\section{Bioengineering RBPs}

Bioengineering RBPs is an important potential tool to control phage host range in biomedical applications. A broader host range has been engineered through recombination of homologous RBPs, whose $\mathrm{C}$ terminus often contains the receptor-binding domain. For instance, recombining the RBP $\mathrm{C}$ termini of enterobacteria phages $\mathrm{T} 3$ and $\mathrm{T} 7$ expanded the host range of each beyond the sum of the host range of both [46]. Similarly, recombination of RBP domains from the T4-like enterobacteria phages WG01 and QL01 resulted in phage WQD, whose host range includes that of both parent phages as well as several novel hosts [47]. A firm understanding of RBP structure and functioning is thus needed for future engineering efforts. Nevertheless, the fact that merely exchanging the tail fibre of phage T3 with that of Yersinia phage $R$ resulted in $T 3$ gaining the ability to infect the phage $R$ host Yersinia tuberculosis shows the potential of bioengineering the RBP for host-range expansion [46]. In addition to increasing the host range of phages through bioengineered RBPs, plasmids containing foreign RBPs can be spread throughout bacterial populations by transducing phages like T7 [48].

\section{Aspects of Host Range after Entry into the Host Cell Genome Replication}

After entry into the cell, several factors influence phage host range. Phages need adaptations to the molecular mechanisms of the host (e.g., codon usage) and evade intracellular defence systems, like restriction modification (RM) systems, clustered regularly interspaced short palindromic repeats (CRISPR), abortive infection (abi), bacteriophage exclusion (BREX), and 
defence island system associated with restriction-modification (DISARM) [49-51]. Phages have developed myriad ways to counter specific intracellular defences, including anti-CRISPR proteins [52], RM inactivation proteins [53], and the recently discovered phage nucleus that protects Pseudomonas phage 201 2-1 DNA during replication and transcription [54]. General antidefence mechanisms such as the latter phage nucleus might be useful assets to broadhost-range phages. With the ongoing characterization of unknown phage proteins [55], we expect that novel mechanisms to overcome host intracellular defences will be uncovered.

Akin to the diversity in bacterial outer-membrane structures, bacteria can defend against phages by encoding unique transcriptional responses to infection [56]. While broad-host-range phages might thus benefit from specialising transcriptional programs to each host, the opposite is true in phages such as Synechococcus phage Syn9 that infects different environmental isolates, as it showed a uniform transcriptional program despite the different responses in three hosts [56]. The same holds for the broad-host-range Bacteroidetes phage $\varphi 38: 1$ that showed a uniform transcriptional program in response to varied transcriptional programs in closely related Bacteroidetes isolates, leading to differences in infection efficiency in those hosts [57]. Phage ب38:1 successfully overcomes intracellular defences and transcriptional responses in its original isolation host, but not in other hosts [58]. These results could indicate that it is difficult for broad-host-range phages to evolve specialised transcriptional programs for different hosts. Still, the conserved aspects of phage transcriptional programs [59] might be selected for in broad-host-range phages.

While phage host range is often discussed in the context of lytic phages, it is a similarly relevant characteristic of temperate phages. The unique challenge for temperate broad-host-range phages is the integrase-mediated integration of their genome into that of their hosts. Integrases rely on distinct target sequences to perform this integration, for example, within conserved tRNA genes [60]. Thus, the process is often host-specific [61], or more precisely it is limited to hosts with the correct integration target site. Brucella phage BiBPO1 is capable of integrating in the genomes of a broad range of hosts by targeting conserved integration sites in tRNA genes [62]. An alternative temperate lifestyle is to circumvent integration altogether by maintaining the genome as a plasmid [63], as was observed in the temperate broad-host-range Ochrobactrum phage POI1126 [64]. Interestingly, the widespread crAssphage does not encode an integrase protein and has never been found integrated in any bacterial genome, but it does have two proteins with putative plasmid replication domains in its genome $[65,66]$. It may thus employ this mechanism to infect diverse hosts, potentially contributing to its widespread abundance.

\section{Exiting the Host Cell}

To complete the lytic life cycle, a broad-host-range phage needs to lyse potentially divergent cell-wall structures and release its progeny. Many phages carry an endolysin which lyses the cells, and a holin which allows the endolysin access to the periplasm at a specific timing in the infection [67]. Endolysins can have lytic host ranges that are broader than the natural host range of the phage [68] and may thus not form critical bottlenecks in the in vivo lytic range. However, the host range may also be bounded by the ability of phage holins to give endolysins access to the cell wall. Moreover, bacteria may suppress phage endolysins to prevent phage progeny from leaving the cell [69], so a broad-host-range phage still needs to possess an altogether compatible lysis system to efficiently exit the various host cells.

\section{Studying Phage Host Range}

As the previous sections show, multiple phages with a broad host range, and their molecular mechanisms, have been identified, and ecological as well as metagenomics studies indicate 
that the broad host range may be more prevalent than previously thought. Yet, a narrow host range is prevalent among isolated and well studied phages. Understanding this disparity demands insight into the evolutionary dynamics of host range (Box 2) and the methodological biases inherent in phage isolation techniques.

\section{The Ecology of Phage Host Range}

In phage-host coevolutionary experiments, an increase in the host range of a phage often coincides with a decrease in the virulence of that phage on each individual host. This trade-off reflects antagonistic pleiotropy in which a mutation is beneficial to one trait but detrimental to a second. Experimental evolutionary studies, for example with Pseudomonas phage $\varphi 6$ [70], have shown evidence of pleiotropic costs to host-range expansion. Examples where such trade-offs are evident include: (i) a recent study of the nontailed Autolykiviridae family of marine Vibrio phages that have broader host ranges but are slower growers than their tailed counterparts [15], (ii) a trade-off between host range and growth rate when comparing enterobacteria phages with different host ranges within the E. coli ECOR strain collection [71], and (iii) a tradeoff between host range and thermostability in polyvalent phage $\lambda$ strains [36]. Antagonistic pleiotropy raises a substantial evolutionary barrier to host-range expansion in the environment, but it is not universal in phage host-range evolution. It has been proposed that, in complex phage-host systems, hosts that evolve resistance to one phage can become susceptible to another phage [72]. For the latter phages, this means that they passively gain infectivity of a new host without incurring mutations or pleiotropic costs [35].

Host density, diversity, and quality are likely relevant factors in determining phage host range, as described in optimal foraging theory [73]. This theory predicts that host-range expansion is favoured only if the potential novel host is either present at higher densities or is of higher quality than the original host. This is illustrated by the example of Pseudomonas phage $\varphi 2$ which expanded its host range only if the density of susceptible hosts was less than $1 \%$ of that of resistant hosts [74]. While optimal foraging phage studies successfully predicted the outcome of competition between narrow- and broad-host-range phages [75], studies with Pseudomonas phage $\varphi 6$ showed that the evolution of generalists depended on both host quality and competition intensity [76]. This may mean that 'broad host range' is selected for in oligotrophic environments, where cell densities are low but species diversity is high [14].

\section{Biases in Experimental Approaches}

Several phage-isolation techniques use monocultures with a high host density, either in liquid or in a double-layer agar medium. These popular assays can detect only those phages that are able to complete a full lytic cycle under the given experimental conditions and within the time frame of the experiment. Moreover, pleiotropic trade-offs between host range and virulence, discussed above, may result in broad-host-range phages with slower growth rates being outcompeted by narrow-host-range phages with higher virulence under these conditions $[15,75]$. Beside these ecological biases, many methods, including double-layer agar and liquid assays, test only a single phage-host association at a time, and only a limited number of phagehost associations in total. These practical considerations mean the actual phage host range might extend to hosts it has not been tested against. Methods such as density gradients, chloroform extractions, and DNA isolations have been shown to contain biases for tailed dsDNA Caudovirales that make up $>90 \%$ of studied phages [15]. Studies have shown that nontailed phage lineages are dominant in some marine environments $[77,78]$, and in the case of nontailed Autolykiviridae, have been found to have a tendency for broad host ranges [15]. Finally, it has been noted that some methods, like spot assays, may overestimate phage host range, meaning that evaluations of phage host range must be treated with some caution [79]. 
Methods are increasingly available that circumvent isolation bias for narrow-host-range phages [80-82]. In particular, methods are being developed to isolate broad-host-range phages infecting different genera. Cocultures of two potential hosts allowed the isolation of phage $\mathrm{SN}-\mathrm{T}$ that formed plaques on hosts from six genera in spot assays [81]. Similarly, sequentially culturing on monocultures of multiple potential hosts resulted in phages that form plaques on multiple strains in the genus Pseudomonas, as well as E. coli, a different host from the class Gammaproteobacteria [82]. Culture-independent techniques, as reviewed in [61], avoid some of these biases, although they come with other biases of their own. Examples include microfluidic PCR [83], phage fluorescent in situ hybridization (FISH) [84], and metagenomic chromosome confirmation capture (Meta3C) [85].

\section{Biases in Computational Approaches}

Computational methods to determine phage host range also have biases. While accurate, identification of matching CRISPR-Cas spacers is limited to detecting host-associations of microbes that contain an active CRISPR system [61]. Another popular technique is detecting similarity in oligonucleotide usage profiles between the genomes of phages and of potential hosts, but this similarity score is a sliding scale, and reliability thresholds remain obscure [1]. Machine-learning techniques hold promise but urgently require diverse and high-quality training data in the form of high-throughput phage-host interaction screens of naturally occurring viruses. The currently known phage-host interactions available in databases remain a limited and, as explained above, a biased subset of the naturally occurring interactions. Due to the sensitivity of machine-learning techniques to training data biases, these techniques should be treated with caution in the face of the diversity of the natural virosphere.

\section{Concluding Remarks}

Three major developments have catalysed the surging interest in bacteriophage research in recent years. First, viruses observed in environmental samples through epifluorescence microscopy [86] and metagenomic screens consistently showed that the majority of natural viruses remain uncharacterized $[12,65]$. Second, discoveries - including CRISPR-Cas - have led to biotechnological innovations [87], adding interest to previously unknown aspects of phage-host interactions. Third, phage therapy seems a promising weapon to combat the global rise of antibiotic resistance in bacterial pathogens [88].

Studying the molecular mechanisms of phage host range and specificity is essential for a wide variety of clinical and industrial applications. We have shown that specificity is a complex notion, dependent on many mechanisms at every step of the phage life cycle. As we continue to find novel phages and study their host interactions, current notions on host range will continue to adapt. The ongoing coevolution between microbes and their viruses has driven a multitude of innovations, and thus novel fascinating discoveries with paradigm-shifting consequences are to be expected (listed in Outstanding Questions). Indeed, the increased interest in phage biology has recently led to discoveries of remarkable molecular mechanisms in phages, including phages forming nucleus- and tubulin-like structures upon infection [89], phages incorporating eukaryotic genes into their genome [90], and phages engaging in quorum sensing [91].

Meanwhile, advanced computational models of phages and their hosts, including their ecoevolutionary dynamics, are needed to integrate our current knowledge on microbial ecosystems and reveal gaps in our understanding [92,93]. Data from recent large-scale environmental surveys, including viral metagenomic sequencing and genome assembly, will enable the integration of phages into ecological models to study the global importance of microbes

\section{Outstanding Questions}

Known broad-host-range phages infect relatively closely related hosts. How taxonomically diverse are the hosts that a single phage can infect?

Recent studies show that host range may be highly variable. How widespread are broad-host-range phages in nature, and what is their ecological impact?

How can broad-host-range phages be incorporated into ecoevolutionary models?

Phage bioengineering is a powerful tool for phage applications such as phage therapy but it requires stability of the phage host range. Can phages with a stable host range be designed?

Several molecular mechanisms for host-range expansion are described in this review. Which other mechanisms remain to be discovered?

How can we use experimental and bioinformatical innovations to overcome biases in phage isolation and host-range assays? 
and their phages, ranging from nutrient cycling to gene flow at scales ranging from the global oceans to the human gut.

\section{Acknowledgments}

We thank A.R. Costa (TU Delft) and T. v. Rossum (TU Delft) for manuscript comments. PAJ and BED were supported by the Netherlands Organization for Scientific Research (NWO) Vidi grant 864.14.004. SJJB was supported by LS6 ERC starting grant 639707, Netherlands Organization for Scientific Research (NWO) VIDI grant 864.11.005, a TU Delft start-up grant and the Netherlands Organization for Scientific Research (NWO/OCW), as part of the Frontiers in Nanoscience program. FLN acknowledges FCT for the grant SFRH/BD/86462/2012.

\section{Supplemental Information}

Supplemental information associated with this article can be found, in the online version, at https://doi.org/10.1016/j.tim. 2018.08.006.

\section{References}

1. Roux, S. et al. (2016) Ecogenomics and potential biogeochemical impacts of globally abundant ocean viruses. Nature 537, 689-693

2. O'Sullivan, L. et al. (2016) Bacteriophage-based tools: recent advances and novel applications. F1000Research 5, 2782

3. Norman, J.M. et al. (2015) Disease-specific alterations in the enteric virome in inflammatory bowel disease. Cell 160, $447-$ 460

4. Manrique, P. et al. (2016) Healthy human gut phageome. Proc. Natl. Acad. Sci. U. S. A. 113, 10400-10405

5. Zablocki, O. et al. (2016) Diversity and ecology of viruses in hyperarid desert soils. Appl. Environ. Microbiol. 82, 770-777

6. Brum, J.R. et al. (2015) Patterns and ecological drivers of ocean viral communities. Science 348, 1261498-1261498

7. Weitz, J.S. et al. (2017) Lysis, lysogeny and virus-microbe ratios. Nature 549, E1-E3

8. Knowles, B. et al. (2016) Lytic to temperate switching of viral communities. Nature 531, 466-470

9. Yu, P. et al. (2017) Suppression of enteric bacteria by bacteriophages: importance of phage polyvalence in the presence of soil bacteria. Environ. Sci. Technol. 51, 5270-5278

10. Modi, S.R. et al. (2013) Antibiotic treatment expands the resistance reservoir and ecological network of the phage metagenome. Nature 499, 219-222

11. Touchon, M. et al. (2017) Embracing the enemy: the diversification of microbial gene repertoires by phage-mediated horizontal gene transfer. Curr. Opin. Microbiol. 38, 66-73

12. Cobián Güemes, A.G. et al. (2016) Viruses as winners in the game of life. Annu. Rev. Virol. 3, 197-214

13. Moebus, K. and Nattkemper, H. (1981) Bacteriophage sensitivity patterns among bacteria isolated from marine waters. Helgoländer Meeresuntersuchungen 34, 375-385

14. Dekel-Bird, N.P. et al. (2015) Host-dependent differences in abundance, composition and host range of cyanophages from the Red Sea. Environ. Microbiol. 17, 1286-1299

15. Kauffman, K.M. et al. (2018) A major lineage of non-tailed dsDNA viruses as unrecognized killers of marine bacteria. Nature 554, 118-122

16. Munson-McGee, J.H. et al. (2018) A virus or more in (nearly) every cell: ubiquitous networks of virus-host interactions in extreme environments. ISME J. 12, 1706-1714

17. Brum, J.R. et al. (2016) Illuminating structural proteins in viral 'dark matter' with metaproteomics. Proc. Natl. Acad. Sci. U. S. A. 113, 2436-2441

18. Paez-Espino, D. et al. (2016) Uncovering Earth's virome. Nature 536, 425-430

19. Siringan, P. et al. (2014) Alternative bacteriophage life cycles: the carrier state of Campylobacter jejuni. Open Biol 4, $130200-$ 130200

20. Cenens, W. et al. (2013) Phage-host interactions during pseudolysogeny. Bacteriophage 3, e25029

21. Peterson, A. (2014) Defining viral species: making taxonomy useful. Virol. J. 11, 131

22. Rosselló-Móra, R. and Amann, R. (2015) Past and future species definitions for Bacteria and Archaea. Syst. Appl. Microbiol. 38, 209-216

23. Serwer, P. et al. (2007) Propagating the missing bacteriophages: a large bacteriophage in a new class. Virol. J. 4, 21

24. Meyer, J.R. et al. (2016) Ecological speciation of bacteriophage lambda in allopatry and sympatry. Science 6056, 1-8

25. Schwarzer, D. et al. (2012) A multivalent adsorption apparatus explains the broad host range of phage phi92: a comprehensive genomic and structural analysis. J. Virol. 86, 10384-10398

26. Tu, J. et al. (2017) Dual host specificity of phage SP6 is facilitated by tailspike rotation. Virology 507, 206-215

27. Dowah, A.S.A and Clokie, M.R.J. (2018) Review of the nature, diversity and structure of bacteriophage recepto binding proteins that target Gram-positive bacteria. Biophys. Rev. 1, 1-8

28. Silva, J.B. et al. (2016) Host receptors for bacteriophage adsorption. FEMS Microbiol. Lett. 363, 1-11

29. Takeuchi, I. et al. (2016) The presence of two receptor-binding proteins contributes to the wide host range of staphylococca twort-like phages. Appl. Environ. Microbiol. 82, 5763-5774

30. Perry, E.B. et al. (2015) The molecular and genetic basis of repeatable coevolution between Escherichia coli and Bacteriophage T3 in a laboratory microcosm. PLoS One 10, e0130639

31. Meyer, J.R. et al. (2012) Repeatability and contingency in the evolution of a key innovation in phage lambda. Science 335 $428-432$

32. Viana, D. et al. (2015) A single natural nucleotide mutation alters bacterial pathogen host tropism. Nat. Genet. 47, 361-366

33. Marston, M.F. et al. (2012) Rapid diversification of coevolving marine Synechococcus and a virus. Proc. Natl. Acad. Sci. U. S A. $109,4544-4549$

34. Le, S. et al. (2013) Mapping the tail fiber as the receptor binding protein responsible for differential host specificity of Pseudomonas aeruginosa bacteriophages PaP1 and JG004. PLoS One 8, $1-8$

35. Schwartz, D.A. and Lindell, D. (2017) Genetic hurdles limit the arms race between Proch/orococcus and the T7-like podoviruses infecting them. ISME J. 11, 1836-1851

36. Petrie, K.L. et al. (2018) Destabilizing mutations encode nongenetic variation that drives evolutionary innovation. Science 359 $1542-1545$

37. Liu, M. (2002) Reverse transcriptase-mediated tropism switching in Bordetella bacteriophage. Science 295, 2091-2094 
38. Tétart, F. et al. (1996) Bacteriophage T4 host range is expanded by duplications of a small domain of the tail fiber adhesin. J. Mol. Biol. 258, 726-731

39. Minot, S. et al. (2012) Hypervariable loci in the human gut virome. Proc. Natl. Acad. Sci. U. S. A. 109, 3962-3966

40. Paul, B.G. et al. (2015) Targeted diversity generation by intraterrestrial archaea and archaeal viruses. Nat. Commun. 6, 1-8

41. Chow, L.T. and Bukhari, A.I. (1976) The invertible DNA segments of coliphages Mu and P1 are identical. Virology 74, 242248

42. Golomidova, A.K. et al. (2016) Branched lateral tail fiber organization in T5-like bacteriophages DT57C and DT571/2 is revealed by genetic and functional analysis. Viruses $8,1-21$

43. Johnson, M.C. et al. (2015) Sinorhizobium meliloti phage ФM9 defines a new group of T4 superfamily phages with unusual genomic features but a common $\mathrm{T}=16$ capsid. J. Virol. 89, 10945-10958

44. Tzipilevich, E. et al. (2017) Acquisition of phage sensitivity by bacteria through exchange of phage receptors. Cell 168, 186199.e12

45. Turnbull, L. et al. (2016) Explosive cell lysis as a mechanism for the biogenesis of bacterial membrane vesicles and biofilms. Nat. Commun. 7, 11220

46. Ando, H. et al. (2015) Engineering modular viral scaffolds for targeted bacterial population editing. Cell Syst. 1, 187-196

47. Chen, M. et al. (2017) Alterations in gp37 expand the host range of a T4-Like phage. Appl. Environ. Microbiol. 83, e01576-17

48. Yosef, l. et al. (2017) Extending the host range of bacteriophage particles for DNA transduction. Mol. Cell 66, 721-728.e3

49. Samson, J.E. et al. (2013) Revenge of the phages: defeating bacterial defences. Nat. Rev. Microbiol. 11, 675-687

50. Goldfarb, T. et al. (2015) BREX is a novel phage resistance system widespread in microbial genomes. EMBO J. 34, 169183

51. Ofir, G. et al. (2018) DISARM is a widespread bacterial defence system with broad anti-phage activities. Nat. Microbiol. 3, 9098

52. Pawluk, A. et al. (2016) Inactivation of CRISPR-Cas systems by anti-CRISPR proteins in diverse bacterial species. Nat. Microbiol. 1, 1-6

53. Rifat, D. et al. (2008) Restriction endonuclease inhibitor $|P|^{*}$ of bacteriophage T4: a novel structure for a dedicated target. J. Mol. Biol. 375, 720-734

54. Chaikeeratisak, V. et al. (2017) Assembly of a nucleus-like structure during viral replication in bacteria. Science 355, 194-197

55. lyer, L.M. et al. (2017) Polyvalent proteins, a pervasive theme in the intergenomic biological conflicts of bacteriophages and conjugative elements. J. Bacteriol. 199, 1-31

56. Doron, S. et al. (2016) Transcriptome dynamics of a broad hostrange cyanophage and its hosts. ISME J. 10, 1437-1455

57. Howard-Varona, C. et al. (2017) Regulation of infection efficiency in a globally abundant marine Bacteriodetes virus. ISME J. 11, 284-295

58. Howard-Varona, C. et al. (2018) Multiple mechanisms drive phage infection efficiency in nearly identical hosts. ISME J. 12, 1605-1618

59. Blasdel, B.G. et al. (2017) Comparative transcriptomics analyses reveal the conservation of an ancestral infectious strategy in two bacteriophage genera. ISME J. 11, 1988-1996

60. Williams, K.P. (2002) Integration sites for genetic elements in prokaryotic tRNA and tmRNA genes: sublocation preference of integrase subfamilies. Nucleic Acids Res. 30, 866-875

61. Edwards, R.A. et al. (2016) Computational approaches to predict bacteriophage-host relationships. FEMS Microbiol. Rev. 40, 258-272

62. Hammerl, J.A. et al. (2016) Analysis of the first temperate broad host range Brucellaphage (BiPBO1) isolated from $B$. inopinata. Front. Microbiol. 7, 1-13
63. Gilcrease, E.B. and Casjens, S.R. (2018) The genome sequence of Escherichia coli tailed phage D6 and the diversity of Enterobacteriales circular plasmid prophages. Virology 515, 203-214

64. Jäckel, C. et al. (2017) Prevalence, host range, and comparative genomic analysis of temperate Ochrobactrum phages. Front. Microbiol. 8, 1-16

65. Dutilh, B.E. et al. (2014) A highly abundant bacteriophage discovered in the unknown sequences of human faecal metagenomes. Nat. Commun. 5, 1-11

66. Yutin, N. et al. (2018) Discovery of an expansive bacteriophage family that includes the most abundant viruses from the human gut. Nat. Microbiol. 3, 38-46

67. Loessner, M.J. (2005) Bacteriophage endolysins - current state of research and applications. Curr. Opin. Microbiol. 8, 480-48

68. Kong, M. and Ryu, S. (2015) Bacteriophage PBC1 and its endolysin as an antimicrobial agent against Bacillus cereus. Appl. Environ. Microbiol. 81, 2274-2283

69. Roces, C. et al. (2016) Reduced binding of the endolysin LYsTP712 to Lactococcus lactis $\Delta \mathrm{ftsH}$ contributes to phage resistance. Front. Microbiol. 7, 1-10

70. Ford, B.E. et al. (2014) Frequency and fitness consequences of bacteriophage $\Phi 6$ host range mutations. PLoS One 9, e113078

71. Keen, E.C. (2014) Tradeoffs in bacteriophage life histories Bacteriophage 4, e28365

72. Avrani, S. et al. (2012) Virus-host swinging party in the oceans Mob. Genet. Elem. 2, 88-95

73. Heineman, R.H. et al. (2008) Optimal foraging by bacteriophages through host avoidance. Am. Nat. 171, E149-E157

74. Benmayor, R. et al. (2009) Host mixing and disease emergence. Curr. Biol. 19, 764-767

75. Guyader, S. and Burch, C.L. (2008) Optimal foraging predicts the ecology but not the evolution of host specialization in bacteriophages. PLoS One 3, e1946

76. Bono, L.M. et al. (2012) Competition and the origins of novelty: experimental evolution of niche-width expansion in a virus. Biol. Lett. 9, 20120616

77. Brum, J.R. et al. (2013) Global morphological analysis of marine viruses shows minimal regional variation and dominance of nontailed viruses. ISME J. 7, 1738-1751

78. Yoshida, M. et al. (2018) Quantitative viral community DNA analysis reveals the dominance of single-stranded DNA viruses in offshore upper bathyal sediment from Tohoku, Japan. Front Microbiol. 9, 1-10

79. Hyman, P. and Abedon, S.T. (2010) Bacteriophage host range and bacterial resistance. In Advances in Applied Microbiology, (1st edn), pp. 217-248, Elsevier

80. Bielke, L. et al. (2007) Salmonella Host range of bacteriophages that infect multiple genera. Poult. Sci. 86, 2536-2540

81. Jensen, E.C. et al. (1998) Prevalence of broad-host-range lytic bacteriophages of Sphaerotilus natans, Escherichia coli, and Pseudomonas aeruginosa. Appl. Environ. Microbiol. 64, 575580

82. Yu, P. et al. (2016) Isolation of polyvalent bacteriophages by sequential multiple-host approaches. Appl. Environ. Microbiol. 82, 808-815

83. Tadmor, A.D. et al. (2011) Probing individual environmenta bacteria for viruses by using microfluidic digital PCR. Science $333,58-62$

84. Allers, E. et al. (2013) Single-cell and population level vira infection dynamics revealed by phageFISH, a method to visualze intracellular and free viruses. Environ. Microbiol. 15, 2306 2318

85. Marbouty, M. et al. (2017) Scaffolding bacterial genomes and probing host-virus interactions in gut microbiome by proximity ligation (chromosome capture) assay. Sci. Adv. 3, e1602105

86. Bergh, Ø. et al. (1989) High abundance of viruses found in aquatic environments. Nature $340,467-468$

87. Makarova, K.S. et al. (2015) An updated evolutionary classification of CRISPR-Cas systems. Nat. Rev. Microbiol. 13, 722-736 
88. Cisek, A.A. et al. (2017) Phage therapy in bacterial infections treatment: one hundred years after the discovery of bacteriophages. Curr. Microbiol. 74, 277-283

89. Chaikeeratisak, V. et al. (2017) Assembly of a nucleus-like structure during viral replication in bacteria. Science 355 , 194-197

90. Bordenstein, S.R. and Bordenstein, S.R. (2016) Novel eukaryotic association module in phage WO genomes from Wolbachia. Nat. Commun. 7, 1-10

91. Erez, Z. et al. (2017) Communication between viruses guides lysis-lysogeny decisions. Nature 541, 488-493

92. Jover, L.F. et al. (2016) Inferring phage-bacteria infection networks from time-series data. R. Soc. Open Sci. 3, 160654

93. Sieber, M. and Gudeli, I. (2014) Do-or-die life cycles and diverse post-infection resistance mechanisms limit the evolution of parasite host ranges. Ecol. Lett. 17, 491-498

94. Cowley, L.A. et al. (2015) Analysis of whole genome sequencing for the Escherichia coli O157:H7 typing phages. BMC Genomics 16,271

95. Endersen, L. et al. (2014) Phage therapy in the food industry. Annu. Rev. Food Sci. Technol. 5, 327-349

96. Schooley, R.T. et al. (2017) Development and use of personalized bacteriophage-based therapeutic cocktails to treat a patient with a disseminated resistant Acinetobacter baumannii infection. Antimicrob. Agents Chemother. 61, e00954-17

97. Soffer, N. et al. (2017) Bacteriophage preparation lytic for Shigella significantly reduces Shigella sonnei contamination in various foods. PLoS One 12, 1-11

98. Drulis-Kawa, Z. et al. (2012) Learning from bacteriophages advantages and limitations of phage and phage-encoded protein applications. Curr. Protein Pept. Sci. 13, 699-722

99. Nobrega, F.L. et al. (2015) Revisiting phage therapy: New applications for old resources. Trends Microbiol. 23, 185-191

100. Chan, B.K. et al. (2013) Phage cocktails and the future of phage therapy. Future Microbiol. 8, 769-783

101. Edgar, R. et al. (2012) Reversing bacterial resistance to antibiotics by phage-mediated delivery of dominant sensitive genes. Appl. Environ. Microbiol. 78, 744-751
102. Yosef, I. et al. (2015) Temperate and lytic bacteriophages programmed to sensitize and kill antibiotic-resistant bacteria. Proc Natl. Acad. Sci. U. S. A. 112, 7267-7272

103. Park, J.Y. et al. (2017) Genetic engineering of a temperate phage-based delivery system for CRISPR/Cas9 antimicrobials against Staphylococcus aureus. Sci. Rep. 7, 1-13

104. Korytowski, D.A. and Smith, H.L. (2014) How nested and monogamous infection networks in host-phage communities come to be. Theor. Ecol. 8, 111-120

105. Flores, C.O. et al. (2011) Statistical structure of host-phage interactions. Proc. Natl. Acad. Sci. U. S. A. 108, E288-E297

106. Gomez, P. and Buckling, A. (2011) Bacteria-phage antagonistic coevolution in soil. Science 332, 106-109

107. Hall, A.R. et al. (2011) Host-parasite coevolutionary arms races give way to fluctuating selection. Ecol. Lett. 14, 635-642

108. Weitz, J.S. et al. (2013) Phage-bacteria infection networks. Trends Microbiol. 21, 82-91

109. Flores, C.O. et al. (2013) Multi-scale structure and geographic drivers of cross-infection within marine bacteria and phages. ISME J. 7, 520-532

110. Roux, S. et al. (2015) Viral dark matter and virus-host interactions resolved from publicly available microbial genomes. eLife 4, e08490

111. Scanlan, P.D. et al. (2013) No effect of host-parasite co-evolution on host range expansion. J. Evol. Biol. 26, 205-209

112. Koskella, B. and Parr, N. (2015) The evolution of bacteria resistance against bacteriophages in the horse chestnut phyllosphere is general across both space and time. Philos. Trans. $A$ Soc. B Biol. Sci. 370, 20140297

113. Vos, M. et al. (2009) Local adaptation of bacteriophages to their bacterial hosts in soil. Science 325, 833-833

114. Hanson, C.A. et al. (2016) Biogeographic variation in host range phenotypes and taxonomic composition of marine cyanophage isolates. Front. Microbiol. 7, 1-14

115. Gurney, J. et al. (2017) Network structure and local adaptation in co-evolving bacteria-phage interactions. Mol. Ecol. 26, 17641777 